\title{
Oral Health During Pregnancy Are We Listening?
}

\author{
David Leader ${ }^{1,2 *}$ \\ ${ }^{1}$ Department of Comprehensive Care, Tufts University School of Dental Medicine, USA \\ ${ }^{2}$ Department of Public Health and Community Medicine, Tufts University School of Medicine, USA
}

*Corresponding author: David Leader, Department of Comprehensive Care, Public Health and Community Medicine Tufts University School of Dental Medicine, Tufts University School of Medicine, USA.

To Cite This Article: David Leader. Oral Health During Pregnancy Are We Listening?. Am J Biomed Sci \& Res. 2019 - 4(6). AJBSR.MS.ID.000847. DOI: 10.34297/AJBSR.2019.04.000847

Received: 嗹 July 17, 2019; Published: 監August 26, 2019

\begin{abstract}
Oral health care during pregnancy benefits the mother and the unborn child. According to the most recent PRAMS data (2017), around 55\% of women in Massachusetts do not receive oral health care in the year before and during pregnancy. This essay describes the disconnect between knowledge and practice and frames the issue as a health communication problem. Health communication theory such as application of the Diffusion of Innovation model may promote adoption of oral health interventions by systemic and oral health providers.
\end{abstract}

Keywords: Beliefs; Education; Gynecologist; Oral Health; Oral Health Knowledge; Dentist; Dental Hygienist; Physician

\section{Introduction}

The Massachusetts Department of Public Health reports that in 2017, 54.1 percent of Massachusetts women had dental prophylaxis in the year prior to pregnancy compared with 56.2 percent during pregnancy. H. Diop [1] PRAMS is the Pregnancy Risk Assessment Monitoring System. It is a survey of maternal experience and attitudes before and after pregnancy conducted by the Centers for Disease Control (CDC) and state public health departments. Dental prophylaxis before and during pregnancy is significant due to the statistical relationship between periodontal disease and pregnancy. Dental treatment during pregnancy is safe and improves maternal oral health. Yet, historic barriers to maternal oral health care during pregnancy continue.

The purpose of this opinion piece is not to argue the science or safety of oral healthcare during pregnancy. Rather, we will shed light on the disconnect between the established science and actual practice. Why do some systemic health providers (physicians, and others) continue to overlook the oral health or advise against oral health care during pregnancy, and why do many dentists and dental hygienists avoid providing treatment during pregnancy?

In 1883, America's first dental journal published the report of a series of opinions of members of the St. Louis Medical Society regarding the safety of tooth extraction during pregnancy [2]. Comments range from the apocryphal tale of draining of an abscess resulting in a miscarriage within a day to extraction of a tooth under generalanesthesialeadingtothepatient'sfirsthealthylivebirth.Some comments advise against providing dental care during pregnancy while others promote making treatment decisions after interview and. While this report appears quaint, the disconnect between knowledge and practice continues.

Recognizing the threat to public health, many state and federal bureaucracies lead efforts to educate healthcare providers and the public regarding the benefit of oral health care during pregnancy [3]. For example, the Massachusetts Department of Public Health convened an interprofessional working group in 2013 to promulgate guidelines for oral healthcare treatment in pregnancy and early childhood. The Commonwealth of Massachusetts published the Massachusetts Oral Health Guidelines for Pregnancy and Early Childhood in 2016. This effort built on policies and guidelines published earlier by groups that include The American College of Obstetrics and Gynecology (ACOG), the National Maternal and Child Health Oral Health Resource Center, the California Dental Association Foundation, the Centers for Disease Control, the Massachusetts Health Quality Partners (MHQP), The National Maternal \& Child Oral Health Resource Center, the New York State Department of Public Health, and the South Carolina Oral Health Advisory Council and Coalition.

Despite the wealth of publications with current, correct advice, incorrect advice continues to exist on medical and dental websites. For example, the American Pregnancy Association's web page, "Pregnancy and Dental Work" recommends delaying routine $\mathrm{x}$-rays until post-partem; this is counter to advice from ACOG and the American Dental Association (ADA) Efforts to promote perinatal 
oral health present classic health communication problems and meet with varying levels of success. Analysis may inform future approaches.

In 2004, a survey of obstetricians and dentists in Bauru and Londrina, Brazil, on their knowledge of prenatal dental care. Surveys designed separately for dentists and for obstetricians. Results demonstrated good current knowledge of prenatal oral health by both groups. However, both groups deviated from what they knew on the side of providing less care or creating barriers. A common barrier is that most dentists preferred to speak with patients' obstetricians prior to providing care even when the dentists and physicians did not think it necessary.

A survey of obstetricians, dentists, and pregnant patients in Franklin County, OH, [4] demonstrates what authors refer to as the "gulf between what is published and what is practiced." Their paper reports the background of recommendations of oral health care during pregnancy as it existed at the time of the survey, 2017. The authors report that although $97 \%$ of dentists surveyed believed that pregnancy patients should receive oral health screening as part of prenatal care, while only $64 \%$ of obstetricians agreed. And while $64 \%$ of obstetricians reported a need for oral care by their patients, less than half include oral health screening questions in their care plan, and only $6 \%$ refer all obstetrical patients to a dentist. Fear of malpractice suit drove nearly all obstetricians and a majority of dentists to delay treatment until after delivery. Only about a fifth of dentists and physicians feel that patients understand the safety and importance of oral health care during pregnancy. Authors advise that this study could be a test of the success of an intervention in the state of Ohio during an effort to improve oral healthcare during pregnancy.

More recently, the department of pediatric dentistry at Tufts University School of Dental Medicine reported on an intervention conducted in the Tufts Medical Center obstetrician/gynecologist (OB/GYN) residency to assess the effect of an oral health educational seminar on residents' knowledge and beliefs. A questionnaire was distributed to 25 residents before, after, and 3 months after the seminar. Recognizing two important limitations, that this study is of low power and does not measure the effect of the improved knowledge, the results of this study suggest that improving oral health care during pregnancy is an innovation that may diffuse throughout medicine and dentistry with appropriately designed interventions [5].

For example, Diffusion of innovations (DI) refers to a health communication model that evaluates the likelihood that the target subjects will adopt a new technique or technology. Additionally, the DI model guides introduction of the innovation to improve the rate of adoption [6].

Characteristics of successful innovations include relative advantage or improvement over the existing technology or technique; compatibility with potential adopters' values, experiences and needs; low complexity; trialability, adopters know that they may try the innovation with low investment; and observability, adopters witness an improvement. DI advises the design of communications that inform potential adopters of these characteristics. For example, a communication with obstetricians may highlight their interest in guiding patients based on evidence. Referral of your pregnant and pre-pregnant patients to a dentist is likely to improve maternal health [7].

Diffusion occurs in phases and will not jump from the first phase to the last. The first step is knowledge, or the introduction of the innovation; dentists may not be aware of the importance of oral healthcare during pregnancy. Persuasion of the target audience involves education such as lectures and publications; some providers may need to see and hear information repeatedly. Members of the target audience must make the decision to apply the innovation or to try it; dentists and physicians must decide to work together to improve women's health during their reproductive years. Implementation of the innovation may require support and reinforcement; reinforcement could be in the form of pay for performance. Finally, the target audience must confirm that they made the correct decision.

The entire target population will not operationalize the innovation at once. DI identifies five categories of adopters, innovators, early adopters, early majority, late majority, and laggards. Innovators will be the first to try a new technique or technology. Interestingly, they are not the target of this type of communication. They are avant guarde, but others are not likely to follow them. Communications that target early adopters and early majority adopters are more successful. This is the segment of the population who are open to new ideas. They will read the research, make a decision, and others will follow their lead. DI suggests that communications target leaders, department heads, and partners in large practices; people identified as evidence-based teachers who are open to new ideas.

\section{Conclusion}

Recently, there has been an evolution in the understanding of the safety and importance of oral health care during pregnancy. State and national, governmental and private organizations agree that oral healthcare before and during pregnancy is advantageous for the mother, fetus and neonate. Dental and systemic health care providers share the responsibility to recommend and improve access to care. Health communication theory, such as the Diffusion of Innovation model, offers a road map to facilitate dissemination and uptake of this public health initiative.

\section{Acknowledgements}

The author would like to thank Dr Hafsatou Diop, Director, Office of Data Translation, State Maternal and Child Health Epidemiologist, PRAMS Director of the Massachusetts Department of Public Health for providing guidance and information. Dr Diop leads the effort to write and disseminate the Massachusetts Oral Health Guidelines for Pregnancy and Early Childhood.

\section{Conflict of interest}

The author discloses that he has close relationships to several women who benefit from improved oral health including his mother, wife, daughters, and daughter-in-law. 


\section{References}

1. (2019) Division of Reproductive Health, National Center for Chronic Disease Prevention and Health Promotion, what is prams?

2. Gorgas FJS (1883) Extraction of teeth in pregnant women. American Journal of Dental Science 16: 328.

3. (2013) Oral health care during pregnancy and through the lifespan Committee Opinion No 569. American College of Obstetricians and Gynecologists 122: 417-422.

4. Strafford K, Shellhaas C, Hade E (2008) Provider and patient perceptions about dental care during pregnancy. J Matern Fetal Neonatal Med 21(1) 63-71.
5. Zanata RL, Fernandes KB, Navarro PS (2008) Prenatal dental care: Evaluation of professional knowledge of obstetricians and dentists in the cities of Londrina/PR and Bauru/SP, Brazil, 2004. J Appl Oral Sci 16(3): 194-200.

6. Shah D, Parsi L, Bagher S, Finkelman M, Loo C (2017) Effectiveness of an oral health education program for obstetrician/gynecologist residents at Tufts Medical Center. J Int Soc Prev Community Dent 7(Suppl 2): S107-S112.

7. Brian O'Connell (2019) What is Diffusion of Innovation and why is it important in 2019? 